Ann. Zootech., I977, 26 (2), 243-253.

\title{
Possibilités de réduction des apports protéiques chez le pintadeau de chair grâce à la supplémentation en lysine et en méthionine
}

\author{
B. LECLERCQ et J. C. BLUM \\ avec la collaboration technique de R. Bonsergent et Solange Guillaumin \\ Station de Recherches avicoles, \\ Centre de Recherches de Tours, I.N.R.A., \\ Nonzilly 37.380 Monnaie (France)
}

\begin{abstract}
Résumé
Deux expériences ont été entreprises afin de savoir dans quelle mesure il est possible de réduire les apports de protéines chez le pintadeau de chair entre 4 et 8 semaines (période de croissance) et 8 et I 2 semaines (période de finition), quand on utilise comme matières premières le mais et le tourteau de soja.

Dans la première expérience le taux protidique varie de I 3,4 à i 8,3 en croissance, les teneurs en lysine et acides aminés soufrés étant maintenus égaux par supplémentation avec de la lysine et de la méthionine purifiées. En finition il en est de même, mais le taux protidique varie de 9,6 à $\mathrm{I} 4,9 \mathrm{p}$. cent.

Irans la seconde expérience, le taux protidique va de $\mathrm{I}_{4,7}$ à I 8,4 p. 100 en croissance, seul l'apport d'acides aminés soufrés étant maintenu constant. En finition, on fait varier le taux protidique de II, I à I 4,7 P. Ioo.

De l'ensemble de ces deux essais on conclut qu'entre 4 et 8 semaines il faut $280 \mathrm{~g}$ de protéines comprenant $13.5 \mathrm{~g}$ de lysine pour que le pintadeau se développe avec une vitesse maximum. La supplémentation en L-lysine ne permet pas d'économiser du tourteau de soja.

De 8 à I 2 semaines l'âge l'apport doit être au minimum de $260 \mathrm{~g}$ de protéines avec $12 \mathrm{~g}$ de lysine. Il faudra accroître l'apport de protéines en le portant à $280 \mathrm{~g}$ lorsque le régime à base de maïs et de tourteau de soja n'est pas supplémenté en lysine purifiée.
\end{abstract}

\section{Introduction}

Nous avons eu l'occasion, au cours d'essais précédents, de constater chez le Pintadeau que les régimes à base de maïs et de tourteau de soja présentaient comme premier facteur limitant les acides aminés soufrés (LECLERCQ, LARBIER et Blum, I975). Par la suite, nous avons précisé les besoins en lysine et en acides aminés soufrés entre les âges de 4 et I 2 semaines (BLum et LeclercQ, I976). Il nous a semblé logique de poursuivre ces études en recherchant le niveau le plus bas que pouvait atteindre le taux protidique des régimes maïs-soja destinés au 
pintadeau lorsqu'une double supplémentation satisfait les besoins en acides aminés soufrés et en lysine. Le résultat obtenu nous a incités à répéter l'expérience avec une simple supplémentation en méthionine.

\section{Matériel et méthodes}

\section{Expérience $n^{\circ} \quad I$}

Elle s'est déroulée en période estivale du I7 juin au 9 septembre r975.

Pour l'ensemble de cette expérience, les animaux sont de souche GaLor (France), élevés selon les conditions décrites précédemment (BLUM, Guillaume et LECLERCQ, I975) à raison de 52 sujets par case. Leur alimentation (régime de démarrage dont la composition figure au tabl. I) est identique jusqu'à l'âge de 4 semaines.

Nous avons comparé 6 régimes en période de croissance (4-8 semaines), ne différant que par leur taux protidique. Leurs teneurs en lysine et en acides aminés soufrés sont maintenues identiques grâce à la supplémentation par la DL-méthionine et le chlorhydrate de L-lysine, de façon à couvrir les besoins tels que nous les avons déterminés. Nous les dénommons I3C, I4C, I5C, I6C, I7C et I $8 \mathrm{C}$, le nombre indiquant le taux protidique théorique, la lettre $C$ signifiant qu'il s'agit de la période de croissance. Ils sont constitués de maîs et de tourteau de soja en proportions variables et fabriqués à partir de deux régimes de base qui sont les régimes extrêmes $(\mathrm{I} 3 \mathrm{C}$ et $\mathrm{I} 8 \mathrm{C})$. Les teneurs réelles en matières azotées totales $(\mathrm{N} \times 6,25)$ déterminées par dosage sont :

$$
\begin{aligned}
& \text { I3C : } 13,4 \text { p. I0o } \\
& { }_{14} \mathrm{C}: 14,3: \text { p. } 100 \\
& \text { I5C : I5,5 p. IOO } \\
& \text { I6C: I6, I p. I00 } \\
& \text { I7C : I7,2 : p. } 100 \\
& \text { I8C : I8,3 p. IOO }
\end{aligned}
$$

Les animaux qui ont reçu ces régimes en croissance sont alimentés par la suite avec un même régime qui est le régime le plus riche de la période de finition.

En effet, 6 autres régimes sont comparés en période de finition (8-I2 semaines). Le principe de constitution de ces régimes est identique à celui de la période de croissance, si ce n'est que les taux protéiques sont plus faibles, ainsi que les teneurs en lysine et en acides aminés scufrés. Il portent la dénomination : $9 \mathrm{~F}$, roF, IIF, $\mathrm{I}_{2} \mathrm{~F}, \mathrm{I}_{3} \mathrm{~F}$ et $\mathrm{I}_{4} \mathrm{~F}$ en fonction de leur taux protidique théorique. Ils sont fabriqués, eux aussi, par mélange en proportions adéquates des deux régimes extrêmes $9 \mathrm{~F}$ et $\mathrm{I}_{4} \mathrm{~F}$ dont la composition fait l'objet du tableau r. Après dosage, les valeurs réelles des taux protidiques sont :

$$
\begin{aligned}
& { }_{9} \mathrm{~F} \quad 9,6 \text { p. Ioo } \\
& \text { IOF I0,7 p. IOO } \\
& \text { IIF II,4 P. IOO } \\
& \text { I } 2 \mathrm{~F} \text { I } 2,5 \text { p. IOO } \\
& \text { I } 3 \mathrm{~F} \text { I3,5 p. } 100 \\
& { }_{14} F \text { I } 4,9 \text { p. IOO }
\end{aligned}
$$

Les animaux mis en expérience pour cette période de finition ont reçu de 4 à 8 semaines le régime $\mathrm{I} 8 \mathrm{C}$. 


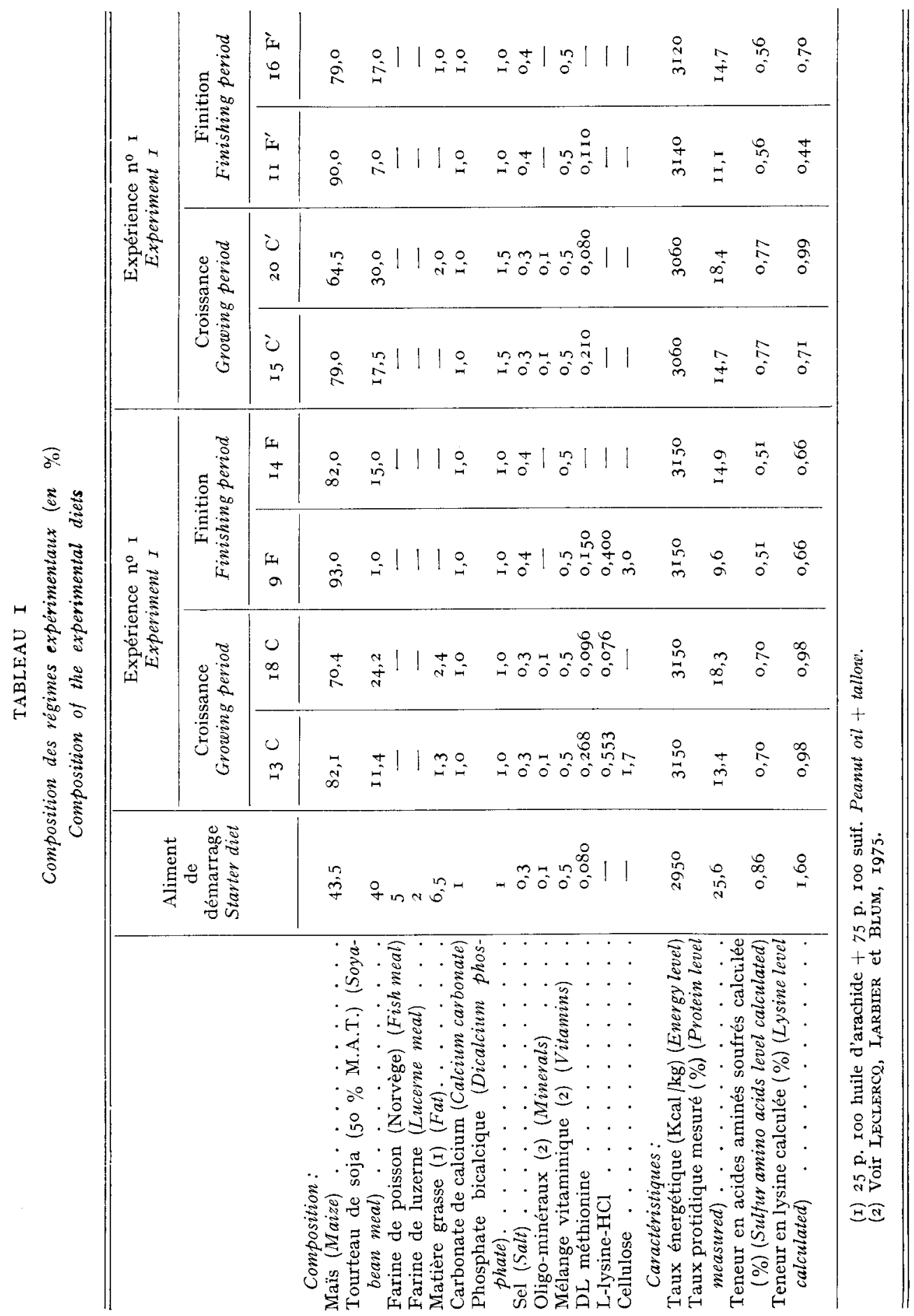




\section{Expérience $n^{\circ} 2$}

Cette expérience a eu lieu du 20 janvier au $\mathbf{3} 3$ avril 1976.

Le protocole de cette expérience ressemble très étroitement à celui de la précédente. La principale différence consiste à n'utiliser que la DL méthionine pour la supplémentation des régimes. Les régimes à base de maîs et de tourteau de soja contiennent les mêmes quantités de minéraux, vitamines, acides aminés soufrés $(0,77 \%)$, calories métabolisables $(3060 \mathrm{Kcal} / \mathrm{kg})$. En période de démarrage (0-4 semaines) les pintadeaux reçoivent la même alimentation. En croissance (4-8 semaines) 6 régimes différents sont distribués : $\mathrm{I}_{5} \mathrm{C}^{\prime}, \mathrm{I}_{6} \mathrm{C}^{\prime}, \mathrm{I}_{7} \mathrm{C}^{\prime}, \mathrm{I} 8 \mathrm{C}^{\prime}, \mathrm{I}_{9} \mathrm{C}^{\prime}$ et $20 \mathrm{C}^{\prime}$. Les valeurs réelles des taux protidiques sont :

$$
\begin{aligned}
& \text { I } 5 \mathrm{C}^{\prime}: \text { I } 4,7 \text { p. IOO } \\
& \text { I6C' : I5,5 p. I00 } \\
& \text { I } 7 \mathrm{C}^{\prime}: \mathrm{I} 6,3 \mathrm{p} \text {. } 100 \\
& \text { I8C' : I7,4 p. I0O } \\
& \text { I } 9 C^{\prime}: \text { I } 8,0 \text { p. Ioo } \\
& \text { 20C' : I8,4 p. IOO }
\end{aligned}
$$

Tous les animaux qui ont servi à l'expérimentation au cours de cette période reçoivent le régime $I 8 \mathrm{C}^{\prime}$ en finition.

Six autres régimes $\mathrm{IIF}^{\prime}, \mathrm{I}_{2} \mathrm{~F}^{\prime}, \mathrm{I}_{3} \mathrm{~F}^{\prime}, \mathrm{I}_{4} \mathrm{~F}^{\prime}, \mathrm{I}_{5} \mathrm{~F}$ et $\mathrm{I}_{6} 6 \mathrm{~F}^{\prime}$ sont comparés entre les âges de 8 et I 2 semaines (finition). Ils assurent les mêmes apports de minéraux, vitamines, calories $(3130 \mathrm{Kcal} / \mathrm{kg}$ ) et d'acides aminés soufrés $(0,56 \%)$. Les valeurs réelles des taux protidiques sont :

$$
\begin{aligned}
& \text { IIF' II,I p. IOO } \\
& \text { I } 2 \mathrm{~F}^{\prime} \text { II,7 p. IOO } \\
& \mathrm{I}_{3} \mathrm{~F}^{\prime} \text { I } 2,5 \text { p. } 100 \\
& \mathrm{I}_{4} \mathrm{~F}^{\prime} \mathrm{I} 3,7 \text { p. } 100 \\
& \mathrm{I}_{5} \mathrm{~F}^{\prime} \mathrm{I} 4, \mathrm{I} \text { p. } 100 \\
& \mathrm{I}_{6 \mathrm{~F}} \mathrm{I} 4,7 \mathrm{p} \text {. } 100
\end{aligned}
$$

Les animaux servant à cette dernière expérimentation ont reçu entre les âges de 4 et 8 semaines un même aliment de croissance qui est le $20 \mathrm{C}^{\prime}$.

Les pintadeaux issus de deux croisements commerciaux (BEGHIN et GALOR) sont répartis en proportions égales dans chaque case, soit au total 48 sujets par case. Chacun des régimes expérimentaux est distribué à 4 cases (4 répétitions). Les pintadeaux portant des bagues, on peut aisément reconnaître le croisement d'origine. Mais ce dernier n'influence guère les résultats obtenus. Il n'en sera donc plus fait mention, étant entendu que nos résultats concernent également les deux croisements.

Dans les deux expériences, les animaux sont pesés individuellement aux âges de 4 , 8 et I 2 semaines. Les aliments, sous forme de granulés, sont pesés aux mêmes âges. Leur composition en acides aminés est calculée à partir des tables de Pion et Fauconneau (I968). 


\section{Résultats}

\section{Expérience $n^{\circ} \quad I$}

Les résultats de l'expérimentation portant sur la période de 4 à 8 semaines figurent dansle tableau 2. Le poids vif croît avec le taux protidique de l'aliment jusqu'à I6,I p. Ioo de protéines. Au-delà, l'amélioration de la croissance, quoique sensible, n'est plus significative. En fait, la différence entre les lots $x 7 \mathrm{C}$ et I8C

\section{TABLEAU 2}

Croissance et consommation alimentaive des pintadeaux en fonction du taux protidique des régimes distribués entre les âges de 4 et 8 semaines (Expérience $n^{0} I$ )

Growth rate and food consumption of the guinea-fowl according to the protein level of the diet fed from 4 to 8 weeks (Experiment $I$ )

\begin{tabular}{|c|c|c|c|c|c|c|}
\hline Lot & I3 C & $\mathrm{I}_{4} \mathrm{C}$ & I $5 \mathrm{C}$ & I6 C & I7 $\mathrm{C}$ & $18 \mathrm{C}$ \\
\hline $\begin{array}{l}\text { Taux protidique réel }(\%) \quad . \quad . \quad . \\
\text { Protein level }\end{array}$ & I 3,4 & 14,3 & I 5,5 & $I 6,1$ & $I_{7}, 2$ & I 8,3 \\
\hline $\begin{array}{l}\text { Poids vif à } 4 \text { semaines (g) . . . } \\
\text { Body weight at } 4 \text { weeks }\end{array}$ & 354 & $35^{2}$ & 357 & 354 & 355 & 359 \\
\hline $\begin{array}{l}\text { Poids vif à } 8 \text { semaines }+2 \text { jours }(\mathrm{g}) \\
\text { Body weight at } 8 \text { weeks }+2 \text { days }\end{array}$ & $804 a\left(^{*}\right)$ & $820 a$ & $890 b$ & $907 \mathrm{bc}$ & $9 \mathrm{I} 6 \mathrm{bc}$ & $93^{8 c}$ \\
\hline $\begin{array}{l}\text { Consommation d'aliment. . . . } \\
\quad \text { (4-8 semaines) (g) } \\
\text { Food consumption (4-8 weeks) }\end{array}$ & I $577 \mathrm{ab}$ & I $4^{8} 5 \mathrm{a}$ & I $588 \mathrm{ab}$ & I $598 \mathrm{ab}$ & I $596 \mathrm{ab}$ & r 6 I $2 b$ \\
\hline $\begin{array}{l}\text { Indice de consommation . . . . } \\
\quad\left(4^{-8} \text { semaines }\right) \\
\text { 1eed conversion ratio }\left(4^{-8} \text { weeks }\right)\end{array}$ & 3,5 oa & $3,17 b$ & $2,98 \mathrm{c}$ & $2,89 \mathrm{~cd}$ & $2,8 \mathrm{gcd}$ & $2,79 \mathrm{~d}$ \\
\hline $\begin{array}{l}\text { Poids vif à I } 2 \text { semaines }(* *) \text {. . } \\
\text { Body weight at r2 weeks }\end{array}$ & I $236 a$ & I $240 a$ & $1289 \mathrm{ab}$ & $1291 \mathrm{ab}$ & I $299 \mathrm{ab}$ & I $333 \mathrm{~b}$ \\
\hline $\begin{array}{l}\text { Indice de consommation } \\
\quad \text { (o-1 } 2 \text { semaines) } \\
\text { Feed conversion ratio (O- } 12 \text { weeks) }\end{array}$ & 3,54 & 3,33 & 3,33 & 3,34 & $3,3 \mathrm{I}$ & 3.23 \\
\hline
\end{tabular}

(*) Les valeurs suivies d'une même lettre ne sont pas significativement différentes au seuil de signification de 5 p. cent.

$(* *)$ Tous les animaux ont reçu le même aliment $\left(x_{4} \mathrm{~F}\right)$ entre 8 et $\mathrm{I}_{2}$ semaines d'âge. All the birds were fed the same diet $\left(I_{4} \mathrm{~F}\right)$ after 8 weeks of age.

paraît accidentelle. Les pintadeaux élevés conjointement pour la mesure du besoin de finition ne pèsent pas plus lourd que les animaux du lot $\mathrm{I}_{7} \mathrm{C}$; ils disposent pourtant du régime $18 \mathrm{C}$ (cf. tabl. 3). L'indice de consommation évolue en sens inverse. Lorsqu'ils reçoivent un régime de finition titrant I4,9 p. Ioo de M.A.T., seuls les deux lots les plus retardés $\mathrm{I}_{3} \mathrm{C}$ et $\mathrm{I}_{4} \mathrm{C}$ ne parviennent pas à compenser le retard de croissance induit par la déficience protéique.

Les régimes expérimentés en finition conduisent aux résultats du tableau 3. Les trois taux protidiques les plus bas retardent légèrement mais significativement la croissance, entraînant une détérioration de l'indice de consommation.

Sur l'ensemble de l'essai, on observe des performances comparables à celles qui sont obtenues en élevage industriel: poids vif à Iz semaines voisin de I 300 grammes, indice de consommation compris entre 3,2 et 3,3 . 
TABLEAU 3

Croissance et consommation alimentaive des pintadeaux en fonction du taux protidique des régimes distribués entre les âges de 8 et 12 semaines (Expévience $n^{0} I$ )

Growth rate and food consumption of the guinea-fowl according to the protein level of the diet fed between 8 and $I 2$ weeks (Experiment $I$ )

\begin{tabular}{|c|c|c|c|c|c|c|}
\hline $\operatorname{Lot}\left(^{*}\right)$ & $9 \mathrm{~F}$ & Io $\mathrm{F}$ & I I F & $12 \mathrm{~F}$ & I $3 \mathrm{~F}$ & $14 \mathrm{~F}$ \\
\hline $\begin{array}{l}\text { Taux protidique réel } \cdot{ }^{*} \cdot \cdot \cdot \cdot \\
\text { Protein level }\end{array}$ & 9,6 & 10,7 & I I , 4 & I 2,5 & 13.5 & 14,9 \\
\hline $\begin{array}{l}\text { Poids vif à } 8 \text { semaines }+2 \text { jours }(\mathrm{g}) \\
\text { Body weight at } 8 \text { weeks }+2 \text { days }\end{array}$ & 917 & 925 & 925 & 920 & 9 I 5 & 9 I 5 \\
\hline $\begin{array}{l}\text { Poids vif à I } 2 \text { semaines }(\mathrm{g}) \text {. . } \\
\text { Body weight at I2 weeks }\end{array}$ & $\begin{array}{l}\text { I }{ }^{\text {I I } 7 a} \\
\left({ }^{* *}\right)\end{array}$ & I $2 \mathrm{IOb}$ & I $257 \mathrm{bc}$ & $1333 d$ & I $28 \mathrm{Icd}$ & I $292 \mathrm{~cd}$ \\
\hline $\begin{array}{l}\text { Consommation d'aliment . . . } \\
\quad(8-12 \text { semaines })(\mathrm{g}) \\
\text { Food consumption }(8-12 \text { weeks })\end{array}$ & I 900 & I 937 & I 920 & I 995 & I 900 & I 929 \\
\hline $\begin{array}{l}\text { Indice de consommation } \cdot \cdot \cdot \text {. } \\
\quad(8-12 \text { semaines }) \\
\text { Feed conversion ratio ( } 8-\mathrm{I} 2 \text { weeks) }\end{array}$ & $9,63 a$ & $6,83 \mathrm{~b}$ & $5,77 \mathrm{c}$ & $4,84 \mathrm{~d}$ & $5,20 d$ & $5,13 d$ \\
\hline $\begin{array}{l}\text { Indice global } \\
\quad \text { (o-I } 2 \text { semaines) } \\
\text { Feed conversion ratio (o-I2 weeks) }\end{array}$ & 3,79 & 3,50 & $3,3^{8}$ & 3,22 & 3,27 & 3,27 \\
\hline
\end{tabular}

(*) Régime démarrage de o à 4 semaines; tégine $18 \mathrm{C}$ de 4 à 8 semaines. Starter diet from o to 4 weeks; diet $18 \mathrm{C}$ from 4 to 8 weeks.

(**) Les moyennes suivies d'une même lettre ne sont pas significativenent différentes au seuil de 5 p. cent.

\section{Expérience $n^{\circ} 2$}

Nous présentons dans le tableau 4 les performances des pintadeaux ayant servi à l'expérimentation entre les âges de 4 et 8 semaines. Les quatre régimes les plus riches permettent des performances excellentes en valeur absolue et remarquablement homogènes. Lorsqu'ils reçoivent en finition un régime riche en protéines ( $\mathrm{I} 8 \mathrm{C}^{\prime}$ ) tous les animaux parviennent au même poids vif à $\mathrm{I} 2$ semaines, dont la valeur dépasse I $300 \mathrm{~g}$ dans la plupart des cas.

Le tableau 5 contient les résultats de l'expérience de finition. Seul le régime le plus pauvre en protéines retarde significativement la croissance du pintadeau et entraîne une dégradation de l'indice de consommation.

Cette dernière expérience se caractérise pour l'ensemble des lots par des valeurs élevées de l'indice de consommation; aucun d'entre eux en effet ne descend en dessous de 3,6 .

\section{Discussion et conclusion}

De l'ensemble de ces deux essais, il ressort clairement que la supplémentation des régimes à base de maïs et de soja par des acides aminés de synthèse permet d'abaisser les taux protidiques des aliments destinés au pintadeau de chair, par rapport à ce qui est pratiqué habituellement. Cependant, une discussion plus précise des résultats est rendue difficile par les variations des quantités d'aliment ingéré, qui se traduisent par des indices de consommation très différents d'un essai à l'autre. Le taux énergétique ne peut, à lui seul, rendre compte de ces diver- 


\section{TABLEAU 4}

Croissance et consommation alimentaire des pintadeaux en fonction du taux protidique des régimes distribués entre les áges de 4 et 8 semaines (Expérience $n^{\circ} 2$ )

Growth rate and food consumption of the guinea-fowl according to the protein level of the diet fed from 4 to 8 weeks of age (Experiment 2)

\begin{tabular}{|c|c|c|c|c|c|c|}
\hline Lot & I $5 C^{\prime}$ & I6 $C^{\prime}$ & $17 C^{\prime}$ & I $8 C^{\prime}$ & I9 $C^{\prime}$ & $20 C^{\prime}$ \\
\hline $\begin{array}{l}\text { Taux protidique }(\%) \\
\text { Protein level }\end{array}$ & I 4,7 & I 5,5 & 16,3 & 17.4 & 18,0 & 18,4 \\
\hline $\begin{array}{l}\text { Poids vif à } 4 \text { semaines }\left({ }^{*}\right)(\mathrm{g}) \\
\text { Body weight at } 4 \text { weeks }\end{array}$ & 334 & $33^{8}$ & $33^{8}$ & 336 & 332 & 327 \\
\hline $\begin{array}{l}\text { Poids vif à } 8 \text { semaines }(\mathrm{g}) \quad . \quad . \quad \\
\text { Body weight at } 8 \text { weeks }\end{array}$ & $\left.8 \mathbf{I}_{4} \mathrm{a}^{(* *}\right)$ & $857 \mathrm{~b}$ & $887 \mathrm{c}$ & $883 \mathrm{c}$ & $88 \mathrm{IC}$ & $878 \mathrm{c}$ \\
\hline $\begin{array}{l}\text { Consommation d'aliment . . . . } \\
\text { (4-8 semaines) (g) } \\
\text { Food consumption (4-8 weeks) }\end{array}$ & I $651 a$ & I $696 \mathrm{~b}$ & I 7 I $2 b$ & r $7 \mathrm{Iob}$ & I $7 \times 7 b$ & I $706 \mathrm{~b}$ \\
\hline $\begin{array}{l}\text { Indice de consommation . . . . } \\
\quad(4-8 \text { semaines }) \\
\text { Feed conversion ratio }(4-8 \text { weeks })\end{array}$ & $3,44 a$ & $3,27 \mathrm{~b}$ & $3, \mathrm{I} 2 \mathrm{C}$ & $3,12 \mathrm{C}$ & $3,13 \mathrm{c}$ & $3, I O C$ \\
\hline $\begin{array}{l}\text { Poids vif à } \mathrm{I} 2 \text { semaines (*) (g) } \\
\text { Body weight at I2 weeks }\end{array}$ & I 290 & I 318 & I 336 & I 326 & I 3 I 5 & I 307 \\
\hline $\begin{array}{l}\text { Consommation d'aliment . . . } \\
\text { (8-I } 2 \text { semaines) }(\mathrm{g})\end{array}$ & 2437 & 2482 & 2447 & 2427 & $24^{\circ} 4$ & 2412 \\
\hline $\begin{array}{l}\text { Food consumption ( } 8-I 2 \text { weeks }) \\
\text { Indice de consommation . . . . }\end{array}$ & $5,12 a$ & $5,3^{8 b}$ & $5,4^{6} \mathrm{bc}$ & $5,49 \mathrm{bc}$ & $5.54 \mathrm{bc}$ & $5,63 \mathrm{c}$ \\
\hline $\begin{array}{l}\text { (8-1 } 2 \text { semaines) } \\
\text { Feed conversion ratio ( } 8-I 2 \text { weeks })\end{array}$ & & & & & & \\
\hline 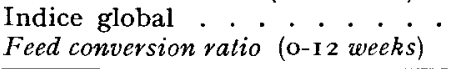 & 3,72 & $3,7^{2}$ & $3,6_{5}$ & 3,65 & 3,67 & 3.68 \\
\hline
\end{tabular}

(*) Alimentation identique pour tous les lots avant l'âge đe 4 semaines (démarrage) et après 8 semaines (régime I $8 \mathrm{C}^{\prime}$ ). The birds were fed the same diets before 4 weeks (starter) and after 8 weeks (I $\left.8 C^{\prime}\right)$.

$\left({ }^{* *}\right)$ Les moyennes suivies d'une même lettre ne sont pas significativement différentes au seuil de 5 p. cent.

\section{TABLEAU 5}

Croissance et consommation d'aliment des pintadeaux en fonction du taux protidique de l'aliment distribué entre 8 et I 2 semaines d'âge (Expérience $n^{\circ} 2$ )

Growth rate and food consumption of the guinea-fowl according to the protein level of the diet fed from 8 weeks of age (Experiment 2)

\begin{tabular}{|c|c|c|c|c|c|c|}
\hline Lot & I I $F^{\prime}$ & I $2 \mathrm{~F}^{\prime}$ & I $3 \mathrm{~F}^{\prime}$ & I $4 F^{\prime}$ & I $5 \mathrm{~F}^{\prime}$ & $16 \mathrm{~F}^{\prime}$ \\
\hline $\begin{array}{l}\text { Taux protidique réel }(\%) \\
\text { Protein level }\end{array}$ & I I , I & I I, 7 & 12,5 & 13,7 & I 4, I & $\mathrm{I} 4,7$ \\
\hline $\begin{array}{l}\text { Poids vif à } 8 \text { semaines (g) (*). } \\
\text { Body weight at } 8 \text { weeks }\end{array}$ & 872 & 869 & 857 & 873 & 868 & 865 \\
\hline $\begin{array}{l}\text { Poids vif à I } 2 \text { semaines }(g) \text {. } \\
\text { Body weight at I2 weeks }\end{array}$ & $\begin{array}{l}\text { I } 201 \mathrm{a} \\
(* *)\end{array}$ & I $256 \mathrm{~b}$ & I $276 b$ & I $293 \mathrm{~b}$ & I $279 b$ & I $283 \mathrm{~b}$ \\
\hline $\begin{array}{l}\text { Consommation d'aliment . . . } \\
\quad \text { (8-12 semaines) (g) } \\
\text { Food consumption (8-12 weeks) }\end{array}$ & $2229 a$ & $2294 \mathrm{ab}$ & $2279 \mathrm{ab}$ & $2353 \mathrm{~b}$ & $233^{6 b}$ & $233^{6 b}$ \\
\hline $\begin{array}{l}\text { Indice de consommation . . . } \\
\quad(8-12 \text { semaines }) \\
\text { Feed conversion ratio ( } 8-12 \text { weeks })\end{array}$ & $6,78 \mathrm{a}$ & $5.92 b$ & $5,45^{\mathrm{C}}$ & $5,60 c$ & $5,68 \mathrm{c}$ & $5.59 \mathrm{C}$ \\
\hline $\begin{array}{l}\text { Indice global } \cdot \cdot \cdot \dot{0} \cdot \dot{\mathrm{o}} 2 \text { weeks) } \\
\text { Feed conversion ratio }\end{array}$ & $3,83 a$ & $3.7 \mathrm{Ib}$ & $3,63 \mathrm{c}$ & $3,67 \mathrm{bc}$ & $3,66 \mathrm{bc}$ & $3,67 \mathrm{bc}$ \\
\hline
\end{tabular}

(*) Régime de dématrage de o à 4 semaines; régimes 20 C' de 4 à 8 semaines. Starter diet from o to 4 reeks; diets $20 C^{\prime}$ from 4 to 8 weeks.

$(* *)$ Les moyennes suivies de la même lettre ne sont pas significativement différentes au seuil de 5 p. Ioo. 
gences. La plus grande part de responsabilité incombe sans doute à la température ambiante. En effet, la première expérience s'est déroulée pendant l'été I975 au cours duquel nous avons enregistré dans le bâtiment d'élevage des températures élevées dépassant parfois $38^{\circ} \mathrm{C}$.

La seconde expérience a eu lieu, elle, en hiver; la température ambiante était presque toujours inférieure à $20^{\circ}$ en dehors des éleveuses. La discussion des résultats exige donc de raisonner non pas sur les teneurs des régimes en protéines et en acides aminés indispensables mais sur les quantités ingérées en cours des périodes étudiées. Ces deux essais peuvent ainsi permettre de préciser la valeur des besoins en protéines totales et en lysine.

\section{I. - Le besoin en protéines du pintadeau}

La figure $x$ illustre les relations qui existent entre les quantités de protéines ingérées entre les âges de 4 et 8 semaines et le gain de poids vif. Quelle que soit la nature de la supplémentation en acides aminés de synthèse, le gain de poids

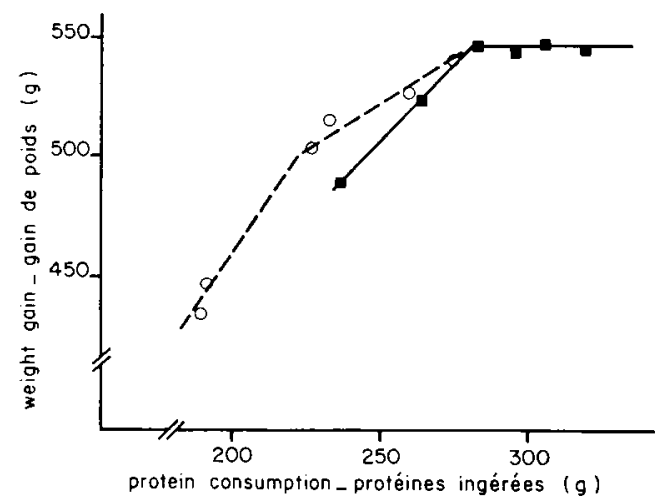

FIG. I. - Relation entre le gain de poids et la quantité de protéines ingérées par le pintadeall entre 4 et 8 semaines (période de croissance).

Relation between weight gain and protein ingested by the guineafowl between 4 and 8 weeks of age (growing period).

$\begin{array}{lll}O-\cdots & \text { Expérience } \mathbf{I} & \text { Experiment } I \\ -\cdots & \text { Expérience } 2 & \text { Experiment } 2\end{array}$

vif maximum est atteint avec $280 \mathrm{~g}$ de protéines. Apparemment, la supplémentation par la lysine ne permet pas d'économiser des protéines. En effet, en dessous du besoin les droites qui lient le gain de poids aux protéines ingérées convergent et coupent au même point la droite horizontale correspondant à la croissance maximum. On peut penser qu'un autre facteur devient limitant en même temps que la lysine. Les travaux portant sur le pintadeau faisant défaut, il est actuellement difficile de formuler des hypothèses sur l'identité du facteur limitant.

Il en va autrement de la période de finition (fig. 2).Le besoin en protéines est d'environ $280 \mathrm{~g}$ avec le mélange maïs et tourteau de soja supplémenté par la méthionine et $260 \mathrm{~g}$ après la double supplémentation en lysine et méthionine. Pendant cette période, la lysine devient donc nettement le facteur limitant après la méthio- 
nine. Du fait de l'abaissement du taux protidique les protéines apportées par le maiss représentent une fraction plus importante des protéines totales. Ces dernières deviennent donc particulièrement déficientes en lysine. L'usage de la lysine de synthèse conduit ainsi à une économie appréciable de protéines.

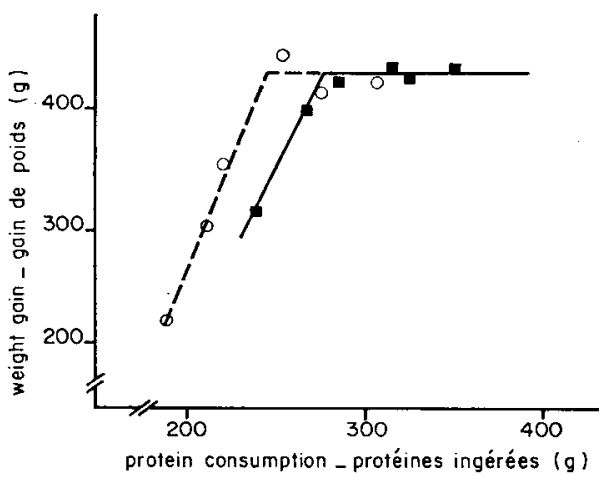

FIG. 2. - Relation entre le gain de poids et la quantité de protéines ingérées par le pintadeau entre 8 et I2 semaines (période de finition). Relation between weight gain and protein ingested by the guineaforel between 8 and I2 weeks of age (finishing period).
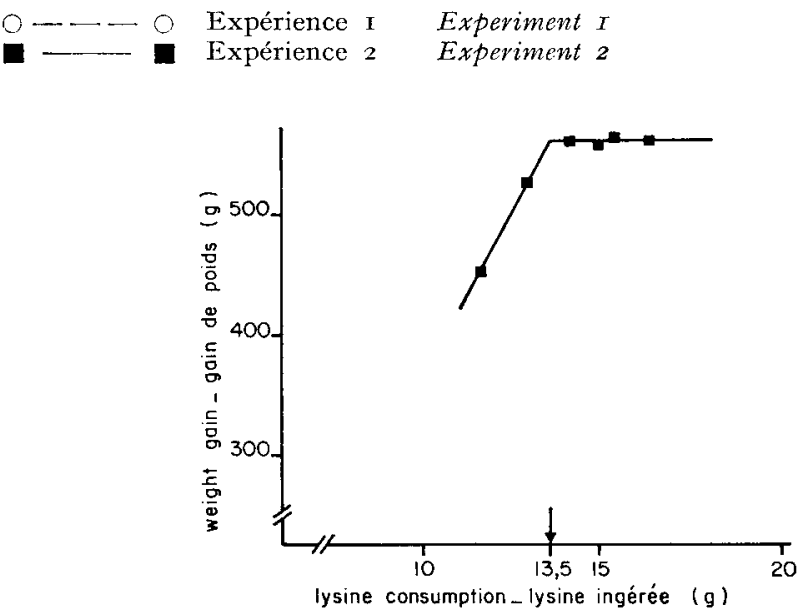

FIG. 3. - Relation établie à partir de l'expérience 2 entre le gain de poids et la quantité de lysine ingérée pav le pintadeau entre 4 et 8 semaines.

Relalion between weight gain and lysine consumption between 4 and 8 weeks of age (experiment 2 )

\section{2. - Le besoin en lysine}

L'expérience $n^{\circ} 2$ au cours de laquelle les proportions de maïs et de tourteau de soja ont varié sans modification des apports d'acides aminés soufrés, permet une estimation du besoin en lysine. Entre 4 et 8 semaines d'âge, le besoin en cet acide aminé estimé d'après les présents résultats est de $\mathrm{I} 3,5 \mathrm{~g}$ (fig. 3). Cette valeur apparaît un peu inférieure à la quantité de $\mathbf{1 4}, 3 \mathrm{~g}$ nécessaire à la croissance maxima 
lors d'essais précédents (BLum et LecLercQ, I976). La différence peut être liée à la composition des régimes : matières premières dissemblables, variabilité de la teneur est de la disponibilité des acides aminés. En finition, les résultats exposés ci-dessus sont en accord avec ceux obtenus par nous-mêmes dans une publication antérieure, soit I2 $\mathrm{g}$ de lysine (fig. 4).

En conclusion, les expériences qui font l'objet de cette publication fournissent une évaluation précise du besoin de protéines et en lysine du pintadeau. L'expression en quantités totales nécessaires à chaque période de croissance est la seule réellement satisfaisante, compte tenu de la variabilité de la consommation. Il faudra adapter la composition des régimes aux conditions d'élevage qui in fluen-

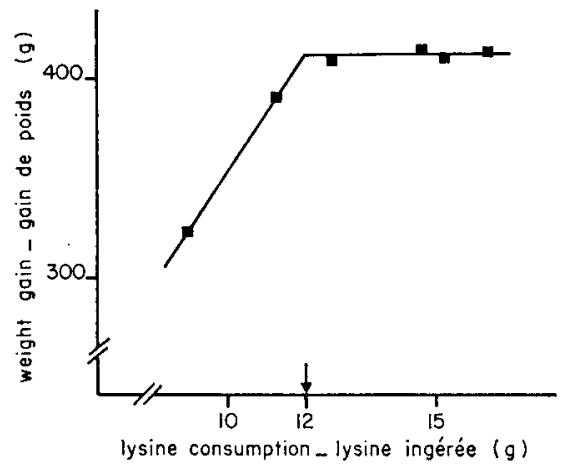

FIG. 4. - Relation établie à partiv de l'expérience 2 entre le gain de poids

et la quantité de lysine ingérée par le pintadeau entre 8 et iz semaines.

Relation between weight gain and lysine consumption between 8 and I2 weeks of age (experiment 2 )

cent le niveau d'ingestion d'aliment de façon à ce que l'oiseau y trouve ce qui est indispensable à sa croissance. Nos résultats permettent en outre de préciser les épargnes de protéines réalisables grâce à l'emploi de la méthionine et de la lysine de synthèse.

Recu pour publication en janvier 1977.

\section{Summary}

Possibilities of reducing protein levels in the diets of guinea-fowl broilers owing to lysine and methionine supplementation.

Two experiments were conducted in order to investigate the possibilities of reducing protein levels in the diets formulated for guinea-fowl broilers, between either 4 and 8 weeks of age (growing period) or 8 and I 2 weeks (finishing period). The main ingredients of each diet were maize, soyabean meal and fat.

In the first experiment the protein level varied from I 3.4 to 18.3 per cent during the growing period but constant levels of lysine and total sulphur amino acids were maintained by the addition of L-lysine and DL methionine. During the finishing period the protein level varied from 9.6 to $\mathrm{I}_{4.9}$ per cent and the concentration of lysine and sulphur amino acids were the same in all diets.

In the second experiment the protein level varied from I 4.7 to I 8.4 per cent during the grow- 
ing period, while the concentration of sulphur amino acids only remained constant. It was the same in the finishing period but the protein level varied from II.I to I 4.7 per cent.

From both these experiments it is concluded that $280 \mathrm{~g}$ of protein and $\mathrm{I} 3.5 \mathrm{~g}$ of lysine are needed by the guinea-fowl for maximum growth from 4 to 8 weeks of age; the lysine supplementation is not able to partly replace protein (fig. I).

During the finishing period the requirements for protein and lysine are respectively $260 \mathrm{~g}$ and $12 \mathrm{~g}$. Nevertheless it is necessary to give $280 \mathrm{~g}$ of protein if the lysine supplementation is omitted (fig. 2).

\section{Références bibliographiques}

Blum J. C., Guillaume J., Leclerce B., 1975. Studies of the energy and protein requirements of the growing guinea fowl. Brit. Poult. Sci., $x 6,137^{-168 .}$

Blum J. C., Leclercq B., 1976. Besoins du pintadeau de chair en lysine et en acides aminés soufrés pendant les périodes de croissance et de finition. Ann. Zootech., 25, 397-406.

Leclerco B., Larbier M., Blum J. C., I975. Emploi de la DL-méthionine et de la L-lysine $\mathrm{HCl}$ pour réduire l'apport alimentaire de protéines chez le pintadeau de chair. Ann. Zootech., $24,229-235$.

Pion R., Fauconneau G., ig68. Les ressources mondiales actuelles et futures en protéines disponibles pour l'alimentation de l'homme. Leurs possibilités d'utilisation. in 'Isotope studies on the nitrogen chain" International Atomic energy Agency Vienna. 